\title{
Uma Infra-Estrutura de Apoio a um Processo de Medição de Projetos em Micro e Pequenas Empresas de Software
}

\author{
Gustavo M. Rocha, Leonardo A. Ciscon \\ Instituto de Ciências Exatas - Universidade Federal de Minas Gerais (UFMG) \\ CEP: 31270-010 - Belo Horizonte - MG - Brasil \\ \{gustavom, ciscon\}@dcc.ufmg.br
}

\begin{abstract}
Micro and Small Enterprises (MSE) represent $77 \%$ of software companies in Brazil. However, about $96 \%$ get out of business during their first two years after establishment. This high level of mortality is mainly by the lack of quality assurance of its products where the quality of the final product is largely determined by its production process. One of the factors of success in improving the software production process is the presence of measuring activities. Based on these facts this work presents an MSE focused measuring infrastructure that consists of a measuring process (PROMPES) and a tool to support the process (FAM). This work also report a case study conducted with the application of this infrastructure in a small software company.
\end{abstract}

Resumo. As micro e pequenas empresas representam $77 \%$ das empresas de software brasileiras. Entretanto, cerca de $96 \%$ encerram suas atividades antes de completarem dois anos de vida. A alta taxa de mortalidade se dá principalmente pela falta de garantia da qualidade de seus produtos sendo que, a qualidade do produto final é, sobretudo, determinada pelo seu processo produtivo. Um dos fatores determinantes na melhoria do processo produtivo de software é a presença de abordagens de medição. Assim, este trabalho apresenta o processo de medição PROMPES e a ferramenta de apoio a esse processo, FAM. Este trabalho relata ainda um estudo de caso realizado com a aplicação do PROMPES e da FAM em uma pequena empresa de software.

\section{Introdução}

Atualmente, a indústria de desenvolvimento de software é uma das que crescem mais rapidamente no setor de Tecnologia da Informação (TI) no Brasil [Wangenheim et al., 2006a]. Diversas empresas atuam neste mercado e de acordo com dados apresentados pelo Ministério de Ciência e Tecnologia [MCT, 2005], as Micro e Pequenas Empresas de Software (MPEs - caracterizadas como empresas com menos de 50 empregados) representam $77 \%$ da força de trabalho do mercado de software brasileiro. Contudo, o número de MPEs que encerram suas atividades antes de completarem os dois primeiros anos de vida é bastante elevada (cerca de 96\%) e segue uma tendência também observada em outros setores da economia [SEBRAE, 2004].

Um dos fatores que contribuem para este elevado nível de mortalidade é a falta de garantia da qualidade de seus produtos sendo que a qualidade do produto final em uma organização é determinada principalmente pelo seu processo produtivo [Weber et al., 2005]. Segundo dados do MCT [MCT, 2005], as MPEs possuem geralmente um 
processo de software informal e, conseqüentemente, dependente principalmente da competência das pessoas envolvidas. Segundo, Thiry e outros [Thiry et al., 2006] a melhoria de produtos através do estabelecimento sistemático de processos pode contribuir significativamente para aumentar a competitividade e as chances de sobrevivência de MPEs.

Pressman [Pressman, 2006] afirma que sem informações quantitativas e qualitativas a respeito de todo o processo de desenvolvimento de software e do produto por parte de uma organização, é impossível tirar qualquer conclusão sobre de que forma está evoluindo a qualidade do produto. Com base neste problema, processos de medição ganharam uma atenção especial tornando-se parte necessária para as organizações que desenvolvem software [Mcgarry et al., 2001].

Para a captura de informações qualitativas e quantitativas dos projetos e estabelecimento sistemático de processos, importantes abordagens e modelos têm sido utilizados. Duas destas abordagens e um destes modelos destacam-se na literatura: GoalQuestion-Metric (GQM) [Basili, 1992], [Basili et al., 1994b], [Basili et al., 1994a], Goal-Driven Software Measurement (GDSM) [Park et al., 1996] e Practical Software Measurement (PSM) [Mcgarry et al., 2001].

Contudo, estas abordagens e modelos foram definidas na forma de modelos genéricos, de difícil aproveitamento por parte das Micro e Pequenas Empresas de software [Anacleto, 2001]. Assim, pelo fato das MPEs representarem um importante papel na economia atendendo mercados onde tipicamente as grandes organizações não atuam [Wangenheim et al., 2006a], e diante da relevância do uso de processos de medição na melhoria de processo e do produto de software, observou-se a necessidade de estabelecer um processo de medição de mais fácil aplicação e aproveitamento por parte das MPEs.

Com base nisso, o objetivo principal do trabalho foi a especificação e $o$ desenvolvimento de uma infra-estrutura de suporte a processos de medições. Esta infraestrutura consiste de dois componentes principais: um processo de medição denominado PROMPES - Processo de Medição para micro e Pequenas Empresas de software e uma ferramenta de suporte denominada FAM - Ferramenta de Apoio às Medições.

Além da definição do PROMPES e da implementação da ferramenta FAM, foi realizado um estudo de caso em uma pequena organização de software. Os resultados obtidos apontaram que grandes benefícios, como um programa de baixo esforço e o melhor monitoramento e acompanhamento dos projetos através das métricas, foram alcançados.

A seção 2 apresenta uma revisão de literatura a cerca da medição e garantia da qualidade em MPEs. A seção 3 apresenta o processo PROMPES e suas características. A seção 4 descreve uma visão geral das principais funcionalidades da ferramenta FAM. A seção 5 apresenta a aplicação do PROMPES e FAM em uma pequena empresa de software. A seção 6 descreve os resultados obtidos e a seção 7 as conclusões deste trabalho. 


\section{Medição e garantia da qualidade em MPEs}

A área de medição tem provado ser uma necessidade essencial para o controle e qualidade dos projetos de software. Apesar das medições serem aplicadas em várias áreas da ciência, elas têm se mostrado de difícil implementação no domínio de software e especialmente no contexto das MPEs por causa de suas limitações [Wangenheim et al., 2003].

Avaliações realizadas em MPEs relatam que a área de processo com a menor aderência ao modelo CMMI é a relacionada com Medição e Análise [Teodora \& Izaskun, 2008]. O trabalho de Cukier [Cukier, 2008] confirma esta falta de aderência apresentando resultados do método de avaliação SCAMPI A, B/C em 18 pequenas e médias empresas.

Apesar das dificuldades discutidas e apresentadas, alguns trabalhos da literatura apresentaram interessantes resultados na aplicação de processos de medição no contexto das MPEs.

No trabalho de Diaz-Ley e outros [Diaz-Ley et al., 2007a] foi apresentada a experiência na definição e implementação de um programa de medição para pequenas e médias empresas de software. O programa proposto (MIS-PyME) [Diaz-Ley et al., 2007b] foi baseado no GQM e possui como principais vantagens a cobertura de características específicas das pequenas e médias empresas de software. Não foi encontrado neste trabalho um detalhamento de quais os critérios usados para a validação do programa proposto. Os resultados apresentados foram baseados em termos qualitativos das percepções do implementador do MIS-PyME e em uma compilação de lições aprendidas na aplicação de programas de medição para pequenas e médias empresas de software.

Trabalhos de Anacleto e outros [Anacleto \& Wangenheim, 2002] e [Anacleto et al., 2002] discutem a mensuração em MPEs no que se refere à gerência de projetos. Foi desenvolvido um modelo de mensuração baseado na abordagem GQM e realizado um estudo de caso planejado em três microempresas de software e executado somente em uma. Como estratégia de validação da proposta apresentada, foi realizada uma análise do custo $\mathrm{x}$ benefício em se aplicar e personalizar programas de mensuração em MPEs e o resultado obtido foi que a execução de mensuração no contexto das MPEs se mostrou de grande eficiência, pois gerou vários benefícios que são do interesse da empresa, com um custo baixo para a mesma. Para execução do modelo proposto foi reportado pelos autores um total de 38 horas.

O trabalho apresentado por Wangenheim e outros [Wangenheim et al., 2003] foca principalmente na estratégia de transformar o GQM em um modelo mais aderente às necessidades das MPEs, focando principalmente no reuso de atividades. A abordagem criada recebeu o nome de GQM LightWeight. A abordagem foi aplicada em 5 MPEs (com 2 a 10 empregados) em projetos de sistemas de gerenciamento de informação. Os critérios para verificação da abordagem proposta foram: aplicabilidade, redução de tempo e esforço (por causa do reuso) e habilidade de disseminar os conceitos de medição.

O trabalho de Garcia e outros [Garcia et al., 2004] apresenta a aplicação da área de Medição e Análise do CMMI no contexto de uma micro e pequena empresa. Não foi 
detectado se foi utilizada alguma abordagem ou modelo de mensuração de software. A completude da proposta foi justificada utilizando o SCAMPI A, um método de avaliação do CMMI para certificar que determinada área está implementada e institucionalizada na organização. Algumas lições foram retiradas da aplicação da área de medição podendo destacar que as mudanças em micro e pequenas organizações parecem ser mais rápidas do que em grandes organizações.

Estes trabalhos não fornecem uma sistemática de aplicação de processos com artefatos, papéis, entradas e saídas bem definidas nem tão pouco fornecem uma ferramenta específica para aplicação de um processo de medição voltado para o contexto das MPEs.

\section{O Processo PROMPES}

O PROMPES além de observar aspectos específicos das micro e pequenas organizações de desenvolvimento de software PROMPES [Revankar et al., 2005], [Teodora \& Izaskun, 2008], [Herndon \& Salars, 2005] e [SEBRAE, 2004], observa também os principais modelos de referência tais como CMMI [SEI, 2002] e MPS.BR [Softex, 2007], o modelo PSM [Mcgarry et al., 2001], a norma ISO/IEC 15939 [ISO/IEC 15939, 2007], a técnica de modelagem de processos de negócio utilizando UML proposta por Eriksson e Penker [Eriksson \& Penker, 2000].

Para facilitar a aplicação de medição neste setor o PROMPES é baseado na abordagem PSM [Mcgarry et al., 2001] que tem sido largamente aceito e implantado na indústria de software ao redor do mundo [Card, 2003b]. O PSM foi selecionado, dentre outros aspectos, em função de fornecer um guia baseado em experiências de como definir e implementar um processo de medição orientado à informação para projetos de software.

Basili e outros [Basili et al., 2007] afirmam que modelos de mensuração, como PSM e GQM, não fornecem uma ligação entre o programa de mensuração e os objetivos de negócio da organização. Esta lacuna dificulta a aplicabilidade e não contribui para a captura de importantes dados utilizados em decisões estratégicas das organizações.

Decidimos portanto utilizar no PROMPES a técnica de Modelagem de Processo de Negócio (MPN) proposta por Eriksson e Penker [Eriksson \& Penker, 2000]. O papel do MPN no PROMPES é fornecer a visão estratégica da organização e assim realizar a ligação entre medidas e decisões estratégicas, ou seja, o MPN auxiliará na identificação das medidas a serem coletadas por parte das MPEs alinhadas aos objetivos estratégicos, considerando, dentre outros, os processos deficientes da organização.

Nossa escolha foi inspirada em parte pelo trabalho de Goethert e Fisher [Goethert \& Fisher, 2003]. Neste trabalho eles fizeram a ligação entre a missão e as metas estratégicas da organização e as medidas voltadas para aspectos operacionais utilizando o arcabouço do Balanced Score Card (BSC) [Kaplan \& Norton, 1992] e um GQM adaptado. A principal diferença deste com o nosso trabalho relaciona-se com escopo: Goethert e Fisher queriam direcionar a tradução da organização no seu alto nível e definir as medidas relacionadas enquanto que nossa intenção é utilizar o MPN para direcionar o foco de quais medidas devem ser tratadas no contexto de desenvolvimento de software nas MPEs. 
Maiores informações acerca do PROMPES e FAM com todas as descrições podem ser encontradas em [Rocha, 2009].

\subsection{Visão Geral}

A especificação Software Process Engineering Meta-Model (SPEM) patrocinada pelo OMG [OMG, 2008] descreve um metamodelo para processos de software. A fundação Eclipse disponibilizou uma plataforma denominada Eclipse Process Framework (EPF) [EPF, 2009] que obedece ao SPEM. Em função do sucesso das especificações do OMG e das ferramentas proporcionadas pela fundação Eclipse decidimos modelar o PROMPES utilizando o EPF. Não vamos apresentar um tutorial sobre o EPF e consideramos suficientemente intuitivo seu uso para o entendimento das explicações que iremos dar em seguida.

O fluxo principal do processo proposto é apresentado na Figura 1. Este fluxo compreende as seguintes macro-atividades: (i) Definir Estratégia Organizacional, (ii) Modelar Conceitualmente, (iii) Modelar Metas e Problemas, (iv) Planejar Medição, (v) Executar Medição e (vi) Analisar e Documentar Resultados.

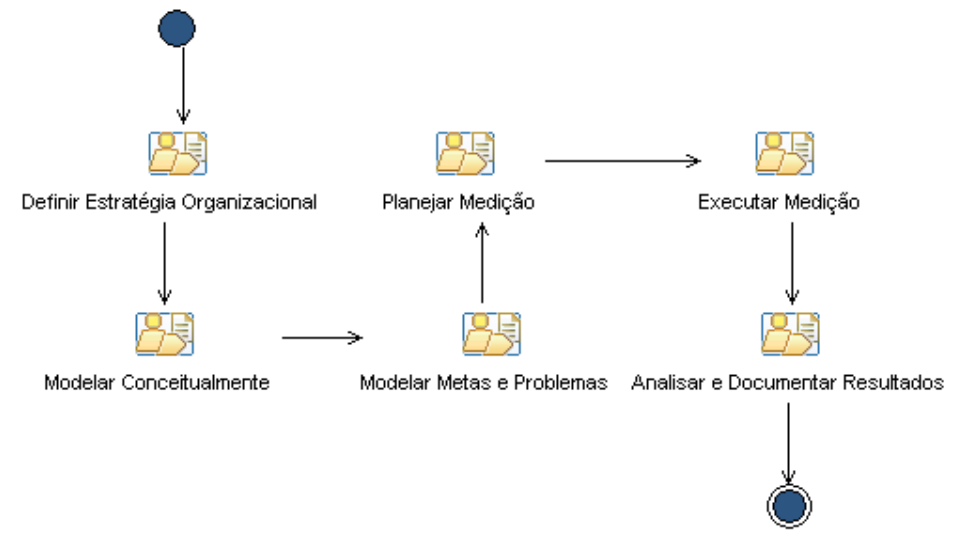

Figura 1 - Fluxo Principal do PROMPES.

As macro-atividades são compostas por atividades, e as atividades do processo são decompostas em etapas. As atividades e etapas do processo foram definidas utilizando os modelos, CMMI, MPS.BR, PSM, ISO/IEC 15939 além de observar as características das MPEs, conforme descrito na seção 3.

A macro-atividade Definir Estratégia Organizacional visa estabelecer e definir como a organização pretende ser vista no futuro através da caracterização da organização em termos de seus negócios. É importante entender seus clientes, concorrentes, a percepção pública da empresa, o ambiente de negócio no qual está inserida, tamanho e posição no mercado, a sua lucratividade e crescimento e o nível de serviço executado. Sendo assim, a estratégia organizacional constitui uma visão integrada do comportamento da organização para que dê suporte ao planejamento da medição [Eriksson \& Penker, 2000].

Desta forma o PROMPES especifica principalmente o entendimento de como a organização funciona através de um elemento da MPN denominado matriz TOWS [Eriksson \& Penker, 2000]. A matriz TOWS é uma ferramenta utilizada para fazer 
análise de cenário organizacional, ou seja, são analisados pontos fortes e fracos internos, oportunidades e ameaças externas e as estratégias de negócio.

A macro-atividade Modelar Conceitualmente define claramente os conceitos importantes envolvidos e utilizados no negócio das MPEs através de um modelo de classes da UML [Eriksson \& Penker, 2000]. Tipicamente como resultado da macroatividade anterior, obtemos a matriz TOWS onde todos os resultados podem ser controlados, bastando que sejam priorizados e mapeados nos processos existentes na organização. Uma vez priorizado, é necessário entender este processo através dos seus conceitos para estabelecer um vocabulário comum entre os envolvidos.

A macro-atividade Modelar Metas e Problemas consiste na modelagem de metas e problemas do processo a ser controlado através de um modelo de atividades da UML [Eriksson \& Penker, 2000]. Os problemas enfrentados pelos processos para atendimentos das metas devem ser definidos e medidos através das métricas para identificação de desvios e erros. Nem sempre os problemas podem ser identificados facilmente, sendo necessário antes definir e medir as metas para somente depois definir os problemas relacionados.

A macro-atividade Planejar Medição procura conhecer as necessidades da organização, para que o emprego do processo de medição possa apontar problemas relevantes e sugerir soluções adequadas. Além disso, é necessário definir quais metas e/ou problemas serão monitorados através das medidas e indicadores.

A macro-atividade Executar Medição procura executar a medição baseado principalmente em um conjunto de medidas previamente estabelecidas. Assim os resultados devem ser guardados, analisados e divulgados para a organização.

A macro-atividade Analisar e Documentar Resultados procura avaliar o processo de medição como um todo, atualizando, identificando e implementando melhorias para que as medidas se tornem mais acuradas e eficazes para a organização em questão.

\section{A Ferramenta FAM}

A ferramenta FAM foi desenvolvida com o objetivo de dar suporte à implementação do PROMPES. Seu principal objetivo é auxiliar no planejamento das medições alinhado aos objetivos de negócio das MPEs.

Baseando-se no método PSM [Mcgarry et al., 2001], a FAM disponibiliza ao usuário todo o suporte ao planejamento das medições e informações sobre as métricas e procedimentos de coleta, armazenamento e análise de dados a serem utilizados.

Já os módulos "Modelar Conceitualmente" e "Modelar Metas e Problemas" foram implementados utilizando uma extensão da ferramenta VisualParadigm [VisualParadigm, 2009]. Esta ferramenta opensource foi utilizada, por permitir que o usuário crie seus modelos UML e por ser de fácil integração com a linguagem C\# e com a ferramenta FAM.

Para apoiar as macro-atividades descritas pelo PROMPES, a FAM: 
- Fornece um mecanismo de definição estratégica com módulos de cadastro e definição do contexto de negócio, declaração da visão e matriz TOWS [Mcgarry et al., 2001];

- Auxilia na modelagem conceitual dos processos realizando o mapeamento das estratégias em processos, e a modelagem visual dos processos através da integração com a ferramenta VisualParadigm;

- Auxilia na modelagem de metas e problemas fornecendo elementos visuais para melhor entendimento das metas relacionadas ao processo a ser medido;

- Fornece um mecanismo de planejamento de medições no nível organizacional e de cada projeto, utilizando como base o método PSM [Mcgarry et al., 2001];

- Auxilia a execução do processo do PROMPES, através da disponibilização de métricas e armazenamento de medidas para cada projeto, formando assim uma base organizacional;

\section{Aplicação do processo PROMPES e ferramenta FAM em uma pequena empresa de software}

O PROMPES e a ferramenta FAM foram aplicados em uma pequena empresa de software criada em 2003, que atua nas áreas de consultoria, desenvolvimento de software e ensino à distância. O quadro de funcionários consiste em 23 colaboradores, alocados em sua maior parte em áreas operacionais. Atualmente, a mesma apresenta projetos e produtos nas áreas de gerência de documentos fiscais, gestão imobiliária, administração escolar, automação comercial e personalização de ferramentas para ensino à distância.

A primeira fase da validação iniciou-se pela escolha da organização em que o PROMPES e a FAM seriam aplicados. O critério usado para essa escolha foi tanto o tamanho da empresa quanto o foco do trabalho, ou seja, empresas de micro ou pequeno porte que possuem como atividade final o desenvolvimento de software

A segunda etapa consistiu na aplicação de uma parte do processo em conjunto com a ferramenta FAM na pequena empresa escolhida. Esta parte do processo aplicado incluía as macro-atividades de Definição Estratégia, Modelagem Conceitual e a Modelagem das Metas e Problemas, contou com a participação de dois Gerentes de Projetos e do Gerente Executivo e a aplicação deu-se em âmbito organizacional.

A terceira etapa consistiu na aplicação do restante do PROMPES em dois projetos envolvendo principalmente os dois Gerentes de Projetos da segunda etapa e o Analista de Medição. Como o autor deste trabalho participou desempenhando o papel de Analista de Medição, não foi necessário a realização de treinamento para aplicação da ferramenta.

Para a definição da macro-atividade Definir Estratégia Organizacional primeiramente levou-se em consideração a definição do contexto do negócio, ou seja, a definição dos clientes, concorrentes, percepção pública da organização, ambiente na qual está inserida, tamanho, lucratividade e nível de serviço. Após esta definição, foi realizada a declaração da visão que consistiu e baseou-se principalmente na política já 
existente onde foram aproveitados aspectos como visão, missão, negócio e valores já definidos e disseminados na organização.

Uma vez caracterizada a organização, foram definidas as estratégias que fazem parte do crescimento da organização utilizando-se da matriz TOWS, conforme apresentado na Figura 2. Esta atividade foi facilitada, pois o representante do papel de Gerente Executivo da organização possuía um bom conhecimento na utilização da matriz SWOT proposta pelo PMBOK [PMI,2005].

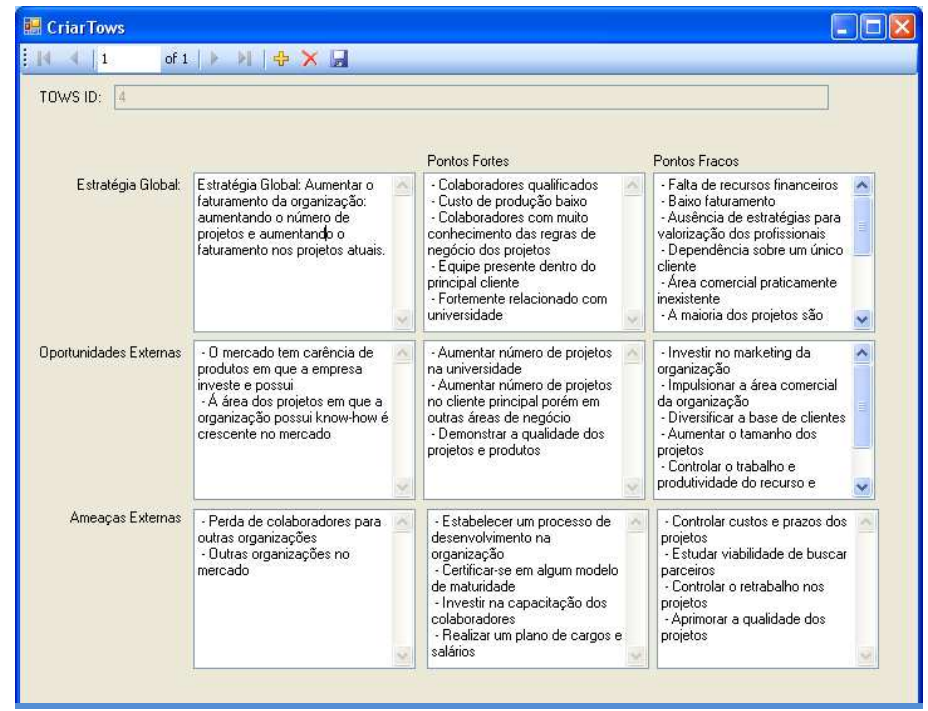

Figura 2 - Interface da FAM para Suporte a Matriz TOWS.

De posse de todas as estratégias, o PROMPES propõe o mapeamento das estratégias de negócio em processos através da execução da macro-atividade Modelar Conceitualmente. Foi realizado um levantamento dos processos de negócio da organização priorizando aqueles que dificultam atingir as estratégias da organização, sejam elas globais ou estratégias de negócio. Os Gerentes de Projeto, em conjunto com o Analista de Medição, agruparam todas as estratégias em processos que devem ser controlados e por sua vez, o Gerente Executivo definiu o processo de Gerenciamento de Projetos como o principal processo que dificulta atender as estratégias de negócio da organização.

Como segunda parte da execução desta macro-atividade, foi definido pelos Gerentes de Projetos, em conjunto com o Analista de Medição, um modelo conceitual para melhor entendimento do processo de Gerenciamento de Projetos, conforme apresentado pela Figura 3 . Esta modelagem baseou-se em um processo de Gerenciamento de Projetos já existente na organização e utilizou-se de um modelo de classes definido pela UML para ilustrar os conceitos envolvidos, estabelecendo assim um vocabulário comum entre os envolvidos. 


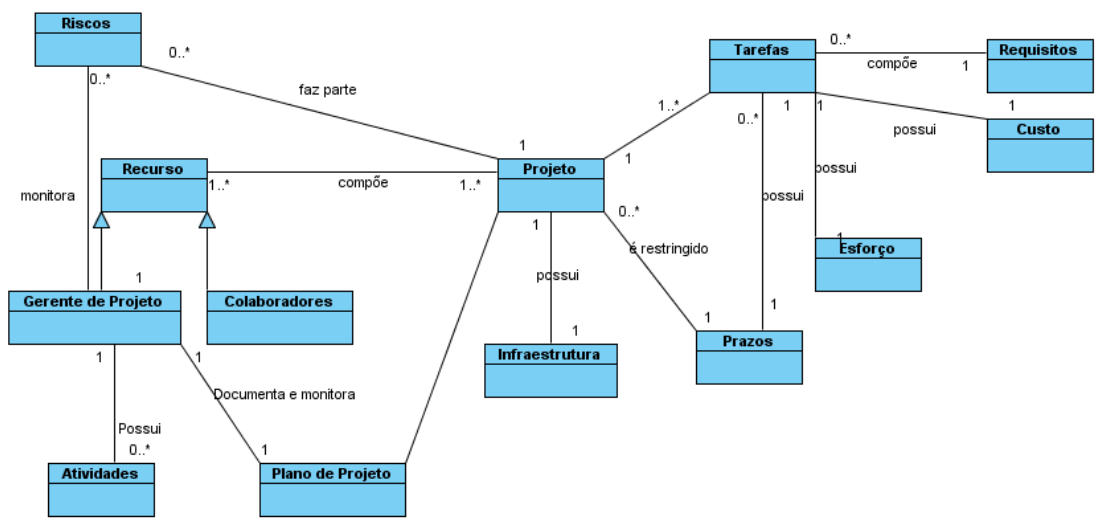

Figura 3 - Modelagem Conceitual do Processo de Gerenciamento de Projetos.

$\mathrm{Na}$ execução da macro-atividade Modelar Metas e Problemas foram modelados recursos que interferem diretamente no processo de Gerenciamento de Projetos. Estes recursos foram classificados como abstrato, objeto de informação e pessoa e modelados conforme apresentado na Figura 4 pela na integração da ferramenta FAM e VisualParadigm. Não foram encontrados recursos físicos que interferiram no processo em questão.

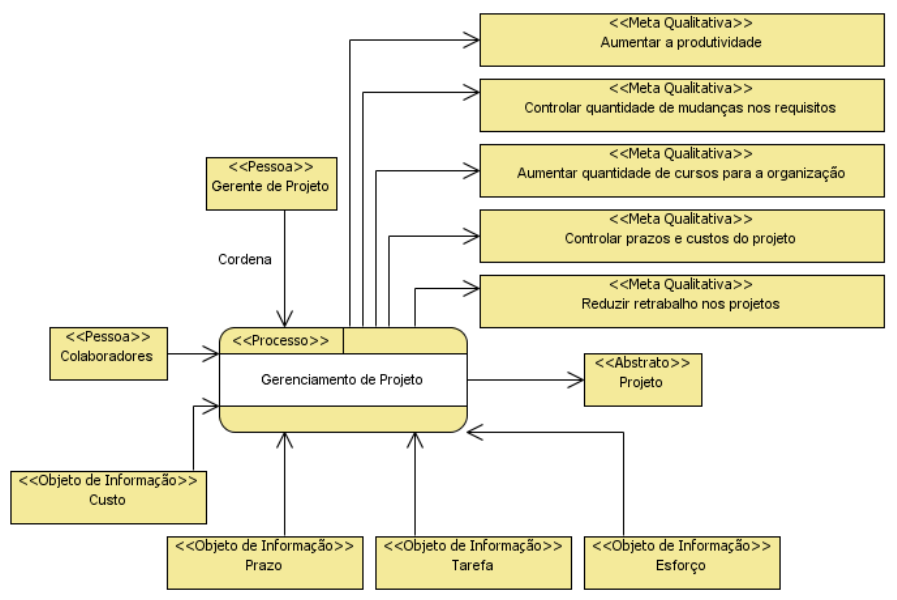

\section{Figura 4 - Definição do Processo de Gerenciamento de Projetos a Ser Controlado.}

Todas as estratégias do processo priorizado de Gerenciamento de Projetos foram derivadas em metas quantitativas, ou seja, que podem ser mensuradas diretamente sem estabelecimento de um critério que transforme uma determinada meta qualitativa em quantitativa. Não foram modelados os problemas para se atingir as metas, pois inicialmente como não se entendia o comportamento das metas a derivação das metas em problemas foi complicado.

Já na execução da macro-atividade Planejar Medição foram caracterizados o contexto de aplicação da medição que consistiu na aplicação da medição em 2 projetos da pequena empresa e alguns procedimentos de coleta. Foi realizada também uma apresentação para todos os envolvidos no processo de medição para assegurar o comprometimento na aplicação do processo em questão. 
Durante o planejamento da medição, as metas priorizadas pelos Gerentes de Projetos foram: Aumentar a produtividade, Controlar custos e prazos e Reduzir retrabalho dos projetos. Foram então definidos, com base nas metas, que os projetos deveriam medir, as seguintes medidas básicas: Produtividade, BCWS (Budget Cost of Work Scheduled), BCWP (Budget Cost of Work Performed), ACWP (Actual Cost of Word Performed) e Esforço de Retrabalho. Estas medidas foram sugeridas pela ferramenta FAM de forma automática através da utilização do PSM.

As medidas sugeridas e definidas anteriormente, foram subdivididas em 3 medidas:

- Medida 1 - Produtividade: foi dividida em duas medidas:

- Medida A: Horas de Implementação;

- Medida B: Tamanho do Requisito (SWPoints);

- Medida 2 - BCWS, BCWP, ACWP: foi dividida em três medidas:

- Medida C: Custo Orçado do Trabalho Agendado;

- Medida D: Custo Orçado do Trabalho Realizado;

- Medida E: Custo Real do Trabalho Realizado;

- Medida 3 - Esforço de Retrabalho: foi realizada apenas uma mudança de nomenclatura:

○ Medida F: Horas de Correção;

Através da FAM, foram cadastradas informações a respeito da identificação das medidas, procedimentos de caracterização (ex: unidade, escala) e por fim informações relativas a forma de periodicidade, registro e coleta das informações, conforme apresentado pela Figura 5.

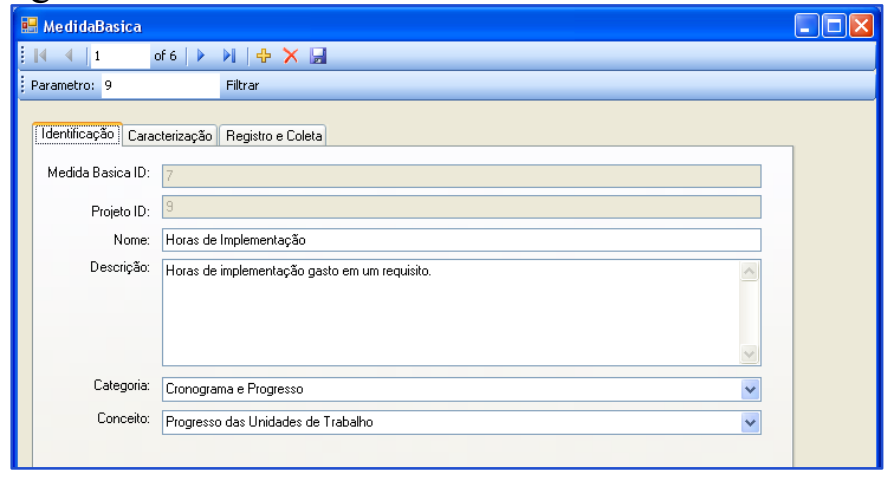

Figura 5 - Interface da FAM para Suporte a Definição das Medidas.

A partir das medidas cadastradas, foram definidos quatro indicadores e suas fórmulas de cálculo para os projetos:

- Indicador A: Produtividade = Horas de Implementação / Tamanho do Requisito;

- Indicador B: Retrabalho = Horas de Correção / Horas de Implementação;

- Indicador C: SPI = Custo Orçado do Trabalho Realizado / Custo Orçado do Trabalho Agendado;

- Indicador D: CPI = Custo Orçado do Trabalho Realizado / Custo Real do Trabalho Realizado.

Através da FAM, foi definido também o momento da geração, como seria a análise dos dados e a forma de distribuição, ou seja, para quais participantes envolvidos 
no processo de medição estes indicadores estariam disponíveis, conforme ilustrado pela Figura 6.

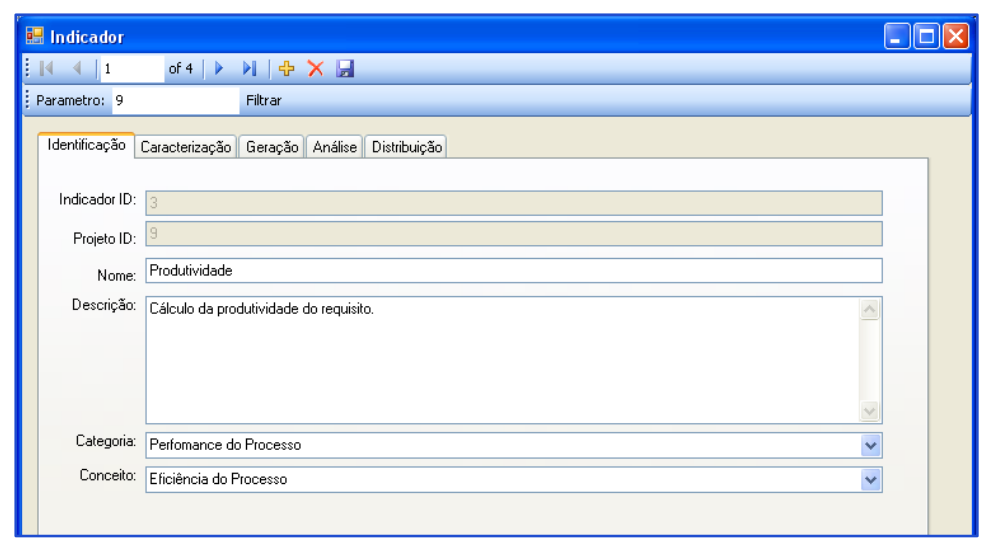

Figura 6 - Interface da FAM para Suporte e Definição dos Indicadores.

A macro-atividade Executar Medição consistiu na coleta, armazenamento, divulgação e análise dos dados de acordo com a especificação de cada indicador. A análise dos dados configurou-se de suma importância para que os Gerentes de Projetos conseguissem identificar desvios ao longo da execução, e assim serem capaz de tomar providências para a mudança de rumo.

Por fim, a macro-atividade Analisar e Documentar resultados consistiu na identificação e implementação de melhorias do PROMPES ao longo da aplicação na pequena empresa. Também foram analisados e avaliados os indicadores, as medidas e o processo de medição como um todo.

A partir dos resultados consolidados podemos observar que os objetivos foram alcançados e os resultados iniciais da aplicação do processo contribuíram para uma análise da situação atual da organização.

\section{Resultados}

A aplicação do PROMPES durou 32 dias, e apesar do baixo tempo de aplicação, vários pontos positivos foram identificados e as vantagens de possuir dados quantitativos para o gerenciamento dos projetos foi bastante assimilada. Assim, espera-se que com o programa de mensuração implantado na pequena empresa consiga principalmente acompanhar e monitorar o andamento dos projetos em relação a prazo, custo, produtividade e retrabalho.

Para verificar a percepção da ferramenta FAM e do processo PROMPES por parte dos integrantes da pequena empresa estudada, foi aplicado um questionário em três integrantes, que atribuíram notas para cada resultado esperado com relação à aplicação do estudo de caso. Tanto a definição das notas como os resultados esperados são definidos pela ISO/IEC 15504 [ISO/IEC 15504, 2004] para que seja verificado a conformidade do processo implantado na organização. O resultado do questionário é apresentado na 1.

Tabela 1 - Notas Médias Atribuídas na Avaliação do Processo de Medição. 


\begin{tabular}{|l|c|c|}
\hline & Resultados Esperados & (L=Largamente, \\
& F=Completamente) \\
\hline REP1 & O comprometimento organizacional é estabelecido e sustentado para \\
implementar as medições? & L \\
\hline REP2 & $\begin{array}{c}\text { As necessidades de informação da organização e dos processos principais } \\
\text { são identificados? }\end{array}$ & F \\
\hline REP3 & Um conjunto apropriado de medidas, guiadas pela necessidade de & F \\
\hline informação são identificados e/ou desenvolvidas? & F \\
\hline REP5 & Astividades de medição são identificadas e executadas? & L \\
\hline REP6 & $\begin{array}{c}\text { Produtos de informação são usados para suportar decisões e prover base } \\
\text { para a comunicação? }\end{array}$ & L \\
\hline REP7 & $\begin{array}{c}\text { O processo de medição e as medidas são avaliados e comunicados para } \\
\text { alimentação do processo? }\end{array}$ & F \\
\hline
\end{tabular}

A consolidação dos resultados mostra que os resultados esperados REP1, REP5 e REP6 foram largamente implementados (L), mas não completamente (F).

Em relação ao REP1, foram percebidos alguns problemas relacionados com a conscientização dos colaboradores em registrarem os dados da forma correta e diariamente. Algumas apropriações eram executadas no dia seguinte e isto ocasionou um pequeno problema principalmente nos indicadores SPI e CPI.

Em relação ao REP5, os Gerentes de Projetos não conseguiram realizar uma interpretação dos dados de forma completa em relação ao indicador de produtividade, pois o mesmo levava em consideração o uso da metodologia própria da organização denominada SWPoints para medir o tamanho do requisito. Foi realizada uma análise superficial deste indicador, porém a interpretação ficou de certo modo um pouco prejudicada.

Em relação ao REP6 o Gerente Executivo considerou que as informações são necessárias para suportar decisões, mas como a aplicação do processo e da ferramenta só considerou dois projetos da organização, não foi possível ainda tomar nenhuma decisão para mudar o rumo ou objetivos de negócio da mesma.

Como o processo de medição PROMPES foi aplicado em um escopo reduzido de 2 projetos, não se pode fazer uma análise detalhada e estatística sobre os benefícios atingidos. À medida que mais projetos forem submetidos à aplicação do PROMPES, espera-se que esta avaliação possa ser melhor realizada.

\section{Conclusão}

No decorrer deste trabalho foram definidos os principais conceitos envolvidos no estabelecimento de programas de medição para MPEs. Assim foi apresentada uma infraestrutura para aplicação do programa de medição nestas organizações. 
Como proposta à resolução de problemas levantados, foi apresentada uma infraestrutura para aplicação de um processo de medição de maneira específica para este tipo de organização. Para isso foi definido o processo PROMPES, automatizado através do desenvolvimento da ferramenta FAM.

A verificação da abordagem definida foi feita através de um estudo de caso aplicando o processo PROMPES e a ferramenta FAM em uma pequena empresa de desenvolvimento de software. No contexto deste estudo de caso os principais resultados foram:

- Apresentação de uma proposta de mensuração personalizada e adaptada para MPEs;

- Baixa personalização no processo original para adequação e aplicação na empresa estudada;

- Apresentação de um programa de baixo esforço e com grandes benefícios para as MPEs;

- Auxílio na percepção da importância e necessidade de modelos de processos, uma vez que as MPEs possuem geralmente um processo de software informal;

- Melhor monitoramento e transparência dos projetos através da mensuração;

- Melhor entendimento por parte dos colaboradores no modelo de negócio da organização;

Como contribuições deste trabalho, pode-se citar:

- Um modelo de medição para aplicação de um programa de mensuração em MPEs com atividades bem definidas;

- Uma ferramenta de apoio às fases de planejamento, coleta e armazenamento dos indicadores;

- Uma nova abordagem de alinhamento das medidas com os objetivos estratégicos das organizações através do uso combinado de PSM e MPN;

\section{Referências}

Anacleto, A. (2001). Modelo de Mensuração para Gerência de Projetos em MicroEmpresas de Software. M.Phil. thesis, Universidade Federal de Santa Catarina, Dezembro, 2001.

Anacleto, A., \& Wangenheim, C. G. V. (2002). Aplicando Mensuração em Microempresas de Software para Suporte da Gerência de Projetos. In: I Simpósio Brasileiro de Qualidade de Software, 2002, Gramado, Brasil.

Anacleto, A., Wangenheim, C. G. V., \& Hammes, J. F. (2002). Mensuração para Suporte da Gerência de Projetos em uma Micro Empresa de Software. In: XIII Conferência Internacional de Qualidade de Software, 2002, Curitiba, Brasil.

Basili, V. R. (1992). Software Modeling and Measurement: The Goal/Question/Metric Paradigm. Tech. rept. UMIACS-TR-92-96. University of Maryland, 1992. 
Basili, V., Caldiera, G., \& Rombach, H. (1994a). The Goal Question Metric Approach. Pages 646-661 of: Encyclopedia of Software Engineering.

Basili, V., Caldiera, G., \& Rombach, H. (1994b). Goal Question Metric Paradigm. Pages 528-532 of: John Wiley \& Sons, Inc (ed), Encyclopedia of Software Engineering, vol. 1.

Basili, V., Heidrich, J., Lindvall, M., Munch, J., Regardie, M., \& Trendowicz, A. (2007). GQM+ Strategies - Aligning Business Strategies with Software Measurement. Pages 488-490 of: I International Symposium on Empirical Software Engineering and Measurement, ESEM, 2007.

Card, D. N. (2003b). Practical Software Measurement. In: XXV International Conference on Software Engineering, 2003, Portland, Oreagon, USA.

Cukier, J. (2008). Problemas de PYMES en el Nivel 2 de Madurez. In: SPEG Latin America, Mar Del Plata, Argentina, 2008.

Díaz-Ley, M., García, F., \& Piattini, M. (2007a). Implementing Software Measurement Programs in Non mature Small Settings. Pages 154-167 of: Lecture Notes in Computer Science, 2007. Lecture Notes in Computer Science, vol. 4895. Springer.

Díaz-Ley, M., G., Carballeira F., \& Piattini, M. (2007b). Software Measurement Programs in SMEs - Defining Software Indicators: A Methodological Framework. Pages 247-261 of: Münch, Jürgen, \& Abrahamsson, Pekka (eds), Lecture Notes in Computer Science, 2007. Lecture Notes in Computer Science, vol. 4589. Springer.

EPF. (2009). Eclipse Process Framework Project (EPF). http://www.eclipse.org/epf/

Eriksson, H., \& Penker, M. (2000). Business Modeling with UML: Business Patterns at Work. John Wiley \& Sons ltd.

Goethert, W., \& Fisher, M. 2003. Deriving Enterprise-Based Measures Using the Balanced Scorecard and Goal-Driven Measurement Techniques. Tech. rept. SEI Software Engineering Institute.

Garcia, S., Cepeda, S., Staley, M., \& Miluk, G. (2004). CMMI as an Improvement Enabler in (at least some!) Small Settings. In: SEI - CMMI Technology Conference and User Group, November, 2004.

Herndon, M., \& Salars, S. (2005). Two Case Studies in Implementing Model Based Process Improvement in Small Organizations. In: First International Research Workshop for Process Improvement in Small Settings, 2005.

ISO/IEC 15504, (2004). ISO/IEC 15504 - Information Technology - Process Assessment.

ISO/IEC 15939. (2007). ISO/IEC 15939 - Information Technology - Software Engineering - Software Measurement Process.

Kaplan, R., \& Norton, D. 1992. The Balanced Scorecard Measures That Drive Performance. Harvard Business Review.

Mcgarry, J., Card, D., Jones, C., Layman, B., Clark, E., Dean, J., \& Hall, F. (2001). Practical Software Measurement: Objective Information for Decision Makers. Addison-Wesley. 
MCT. (2005). A Qualidade e Produtividade no Setor de Software Brasileiro. Tech. rept. $\begin{array}{llll}\text { Ministério de Ciência 2005, } & \text { e Tecnologia, }\end{array}$ http://www.mct.gov.br/index.php/content/view/4495.html.

OMG. (2008). Software Process Engenieering Meta-Model, Version 2.0. http://www.omg.org/technology/documents/formal/spem.htm

Park, R. E., Goethert, W. B., \& Florac, W. A. (1996). Goal-Driven Software Measurement: a $\quad$ Guidebook. http://www.sei.cmu.edu/publications/documents/96.reports/96.hb.002.html: Carnegie Mellon University.

PMI. (2005) A Guide to the Project Management Body of Knowledge (PMBOK® Guide), Third Edition, Project Management Institute.

Pressman, R. S. (2006). Engenharia de Software. Ed. McGraw-Hill, 6ed, 720 p.

Revankar, A., Raghavendr, M., \& Nallagonda, V. M. (2005). Accelerated Process Improvements for Small Settings. In: First International Research Workshop for Process Improvement in Small Settings, 2005.

Rocha, G. M. (2009). Uma Infra-Estrutura de Apoio a um Processo de Medição de Projetos em Micro e Pequenas Empresas de Software. M.Phil. thesis, Universidade Federal de Minas Gerais, Julho, 2009.

SEBRAE. (2004). Fatores Condicionantes e Taxa de Mortalidade de Empresas no Brasil. Tech. rept. Serviço Brasileiro de Apoio às Micro e Pequenas Empresas.

SEI. (2002). CMMI ${ }^{\circledR}$ for Development, Version 1.2. Carnegie Mellon University, Software Engineering Institute, Pittsburgh.

Softex. (2007). Guia de Implementação - Parte 2 - versão 1.1. Disponível em: http://www.softex.br/portal/mpsbr/guias/MPS.BR_Guia_de_Implementacao_Parte_ 2_V1.1.pdf.

Teodora, B., Izaskun, S. (2008). El por qué de la mejora en distintas áreas de las PYMEs. In: SPEG Latin America, Mar Del Plata, Argentina, 2008.

Thiry, M., Wangenheim, C., Zoucas, A., \& Pickler, K. (2006). Uma Abordagem para a Modelagem Colaborativa de Processos de Software em Micro e Pequenas Empresas. In: V Simpósio Brasileiro de Qualidade de Software, 2006, Vila Velha, ES, Brasil.

Visual Paradigm. (2009). Disponível em: www.visual-paradigm.com/.

Wangenheim, C., Punter, T., \& Anacleto, A. (2003). Software Measurement for Small and Medium Enterprises. In: XII Internacional Conference on Empirical Assessment in Software Engineering, Keele, UK, 2003.

Wangenheim, C., Weber, S., Hauck, J., \& Trentin, G. (2006a) (September). Experiences on Establishing Software Processes in Small Companies. Pages 890-900 of: Information and Software Technology, 2006, vol. 48.

Weber, S., Hauck, J., \& Wangenheim, C. (2005). Estabelecendo Processos de Software em Micro e Pequenas Empresas. In: IV Simpósio Brasileiro de Qualidade de Software, 2005, Porto Alegre, RS, Brasil. 